\title{
Loudness adaptation with modulated stimuli
}

Maaike Van Eeckhoutte, Jan Wouters, Tom Francart

KU Leuven - University of Leuven, Department of Neurosciences, ExpORL, Leuven, Belgium

\section{INTRODUCTION}

2 The loudness of an auditory stimulus, defined as its perceptual strength [1], can 3 change over time even though the physical intensity of the stimulus remains 4 constant, leading to loudness adaptation or enhancement. Simple loudness 5 adaptation is described as a decrease in loudness judgment of a steady auditory stimulus presented monaurally (for a review see [2]). The term simple is used to distinguish from induced loudness adaptation in which loudness adaptation to a steady stimulus occurs in the presence of an intermittent stimulus in the contralateral or ipsilateral ear [2]-[5]. This study only deals with simple loudness adaptation. In many studies simple loudness adaptation was only found for specific stimulus parameters, such as low sensation levels ( $<40 \mathrm{~dB}$ SL), high frequencies ( $\geq 2000 \mathrm{~Hz}$ ), and narrow bandwidths (e.g., pure tones compared to broadband noise). Loudness enhancement, an increase in loudness judgment over time, has been shown to occur for stimuli at high sensation levels [6].

Stimuli that give rise to loudness adaptation are often similar to those commonly used for evoking auditory steady-state responses (ASSRs). These responses are auditory evoked potentials whose frequency components remain constant in amplitude and phase over time [7]. ASSRs are often used in research, and clinically they are used for frequency-specific objective hearing assessments. Typical stimuli for evoking frequency-specific ASSRs are modulated pure tones with carrier frequencies corresponding to the audiometric frequencies of 500 , 1000,2000 , and $4000 \mathrm{~Hz}$. While a modulation frequency of $80-90 \mathrm{~Hz}$ is mostly used in clinical devices, the largest response amplitudes are evoked with a modulation frequency of $40 \mathrm{~Hz}$ and a carrier frequency of $500 \mathrm{~Hz}$ [8]-[10]. Amplitude modulation is often used, as well as mixed modulation, a combination of frequency and amplitude modulation. An advantage of mixed-modulation is 
that the responses are significantly larger than with uniquely frequency- or amplitude-modulated stimuli [8], [11].

It is currently unclear whether the stimuli used for ASSR measurements also cause loudness adaptation. If this is the case, the ASSR amplitude may decrease as well, which might in turn have an impact on the use of ASSRs in clinical practice.

Most simple loudness adaptation experiments have been conducted with pure tones as stimuli. Only Scharf [2], and recently also Wynne [12], investigated more complex stimuli, containing either frequency or amplitude modulation. Scharf did not find any difference in loudness adaptation between pure tones and $50 \mathrm{-Hz}$ frequency-modulated tones, which were sinusoidally modulated between $3900 \mathrm{~Hz}$ and $4084 \mathrm{~Hz}$ at $10 \mathrm{~dB} \mathrm{SL}$. In contrast, loudness adaptation with amplitudemodulated stimuli (two-tone complexes) was reduced if the changes in excitation level in the cochlea, produced by amplitude modulations, occurred only within an auditory filter band. Regardless of the modulation frequency, reduced loudness adaptation was also found in Wynne et al. [12] with amplitude-modulated stimuli having a carrier frequency of $12 \mathrm{kHz}$ presented at $15 \mathrm{~dB}$ SL.

The purpose of this study was to investigate whether sustained, amplitude- and mixed-modulated stimuli, typically used for evoking ASSRs, cause a change in loudness judgment over time. Carrier frequencies of $500 \mathrm{~Hz}$ and $2000 \mathrm{~Hz}$ were chosen to compare low and high frequencies. Furthermore, to investigate possible loudness adaptation as well as enhancement effects, a low sensation level of 30 $\mathrm{dB}$ SL was compared to a high sensation level of $70 \mathrm{~dB}$ SL. An additional aim of this study was to investigate whether the participants' detection thresholds would have an influence on their loudness adaptation behavior.

Stimulus parameters in this study were chosen to enable their use in future experiments involving ASSRs. The different modulation types were chosen because of their relevance for ASSRs. While the difference between high and low carrier frequencies might have been more apparent with a $4000 \mathrm{~Hz}$ high carrier frequency, this would also give rise to smaller ASSR amplitudes. A modulation 
frequency of $40 \mathrm{~Hz}$ was used because larger ASSR amplitudes and larger effects were expected with this modulation frequency [8].

Two experiments were conducted to compare loudness adaptation between modulated and unmodulated sinusoids. In the first experiment, sinusoidally amplitude-modulated sinusoids were used. In the second experiment, mixedmodulated sinusoids were used, containing both amplitude and frequency modulation.

\section{MATERIALS AND METHODS}

\section{Participants}

Fourteen healthy young adults (10 female, 4 male, $22.3+-3.1$ years old) participated in the first experiment, and 15 other healthy young adults (13 female, 2 male, $21.7+-0.9$ years old) participated in the second experiment. All participants provided informed consent in accordance with the declaration of Helsinki, and this project was approved by the ethical committee of the University Hospital of Leuven (UZ Leuven). None of the participants had ever participated in a loudness-related experiment before and all were unpaid volunteers. As determined by otoscopy, all participants had normal, unobstructed external ear canals. Pure tone audiometry on the left ear was performed before each test session with a Madsen Electronics Orbiter 922 audiometer and TDH-39 headset. All participants had normal hearing thresholds, $20 \mathrm{~dB}$ HL or better across all octave frequencies from $125 \mathrm{~Hz}$ to $8 \mathrm{KHz}$, with the exception of two participants who had a threshold of $25 \mathrm{~dB}$ HL at $8 \mathrm{kHz}$.

\section{Apparatus and stimuli}

The experiments took place in a soundproof booth. The software platform APEX 3, developed at ExpORL [13], was used for all experiments. Stimuli were created in Matlab R2013a (The Mathworks, Inc., Natick, MA) and presented to the left ear through an Etymotic Research ER-3A insert phone, connected to an RME Hammerfall DSP Multiface II sound card. The level of each stimulus was 
separately calibrated in dB SPL using a 2cc Brüel \& Kjær coupler. The right ear was plugged in order to minimize background noise and other distractions.

Each stimulus had a carrier frequency of either 500 or $2000 \mathrm{~Hz}$ and was presented at either 30 or $70 \mathrm{~dB}$ SL (sensation level). Three modulation types were used in the experiments: no modulation ('SIN'), sinusoidal amplitude modulation ('SAM'), and mixed modulation ('MM').

SAM stimuli were used in experiment I only and are described by the following formula:

$$
y(t)=\sin \left(2 \pi f_{c} t\right) *\left(0.5+0.5 * \sin \left(2 \pi f_{m} t\right)\right)
$$

where $y(t)$ is the stimulus amplitude at time $t, f_{c}$ is the carrier frequency (500 or $2000 \mathrm{~Hz}$ ), and $f_{m}$ the modulation frequency $(40 \mathrm{~Hz})$.

MM stimuli were used in experiment II only. They contained both amplitude modulation and frequency modulation, and are described by:

$$
y(t)=\sin \left(2 \pi f_{c} t+\frac{0.25}{f_{m}} * \sin \left(2 \pi f_{m} t-\frac{\pi}{2}\right)\right) *\left(0.5+0.5 * \sin \left(2 \pi f_{m} t\right)\right)
$$

where $y(t)$ is the stimulus amplitude at time $t, f_{c}$ is the carrier frequency (500 or $2000 \mathrm{~Hz})$, and $f_{m}$ the modulation frequency $(40 \mathrm{~Hz})$.

Spectra of the stimuli are shown in Figure 1. Stimulus bandwidths were respectively $80 \mathrm{~Hz}$ and $240 \mathrm{~Hz}$ for the SAM and MM stimulus conditions.

In summary, each experiment consisted of $2 \times 2 \times 2$ stimulus conditions: modulated (SAM in experiment I, MM in experiment II) or unmodulated (SIN) stimuli, with a carrier frequency of 500 or $2000 \mathrm{~Hz}$, and presented at 30 or $70 \mathrm{~dB}$ SL. These stimulus conditions will be abbreviated as SIN/SAM/MM-500/2000-30/70. Total stimulus duration for the loudness adaptation experiments was always $302 \mathrm{~s}$, and for hearing threshold determination $1 \mathrm{~s}$.

\section{Procedures}

Hearing thresholds in $\mathrm{dB}$ SPL for each stimulus condition were determined using an adaptive three-interval, three-alternative forced-choice (3AFC) procedure. By 
this procedure, three intervals were played consecutively, with three associated visual cues lighting up on a computer screen. Only one random interval contained the stimulus; nothing was presented in the other intervals. The participant was asked to indicate the number of the interval associated with the stimulus after each three-interval period. The level of the stimulus was adjusted based on a twodown, one-up rule, converging to $71 \%$ correct, with step sizes of 10,5 and $2 \mathrm{~dB}$ after 0, 1 and 3 reversals respectively. After 6 reversals the threshold was calculated as the mean of the last 4 reversals. No feedback was provided to the participant.

After hearing threshold determination, stimuli were presented at 30 or $70 \mathrm{~dB}$ above the hearing threshold. Loudness adaptation was measured with a successive magnitude estimation task. During the course of a $302 \mathrm{~s}$ stimulus, participants had to rate its loudness at specific times by typing a self-chosen number related to the perceived loudness. A red visual cue alerted the participants to their task of typing this number. Loudness judgments were obtained in the form of this number, at $2 \mathrm{~s}$ after stimulus onset and at the end of each $20 \mathrm{~s}$ interval thereafter, for a total of 16 measurements. This was done for each stimulus condition in a random order, with the exception that two conditions with the same carrier frequency were never presented successively, to avoid residual adaptation effects due to stimulation of the same frequency without sufficient adaptation recovery time [14]. Participants were carefully instructed that they were free to choose a scale, to use any positive number, including decimals or fractions, and that the same number could be used multiple times. A zero would mean that the stimulus was inaudible. Moreover, the experimenter reminded the participants of the fact that an infinite range of other numbers lies between two given numbers and that there could be no wrong answers. No numerical examples were given.

\section{Data Analysis}

Similar to [6], [15]-[17], individual loudness adaptation percentages were calculated for each participant and stimulus condition as per the following equation: 
where $E_{0}$ is the number given $2 \mathrm{~s}$ after stimulus onset, and $E_{t}$ the number given for the loudness judgment at time $t$. A positive percentage indicates loudness enhancement, a negative percentage indicates loudness adaptation. A loudness adaptation percentage of $-100 \%\left(E_{t}=0\right)$ indicates that the stimulus became inaudible, and a loudness adaptation percentage of $0 \%\left(E_{t}=E_{0}\right)$ indicates that neither adaptation nor enhancement occurred.

\section{Statistical Analysis}

Outliers were detected by the median absolute deviation or MAD-median rule [18]: a loudness adaptation percentage $X_{p, t}$ at time $t$ of participant $p$ was considered an outlier and removed from further analysis if

$$
\frac{\left|X_{p, t}-M_{t}\right|}{M A D / 0.6745}>2.24
$$

with $M_{t}$ the median of the adaptation percentage across participants at time $t$ of a particular stimulus condition and $M A D$ the median of $\left|X_{1, t}-M_{t}\right|, \ldots,\left|X_{N, t}-M_{t}\right|$, with $N$ the number of participants.

Statistical analysis was performed using R [19]. A linear mixed-effects model was used to fit the combined data of experiment I and II. The model included the following predictors of loudness adaptation: time, the participant's threshold, the modulation type (SIN, SAM or MM), sensation level (30 or $70 \mathrm{~dB}$ SL), and carrier frequency (500 or $2000 \mathrm{~Hz}$ ). These predictors were included in the model if judged to be an improvement according to the log-likelihood by analysis of variance (ANOVA). Interaction effects between predictors were also considered. The participant's intercepts and slopes were included as a random effect of the model. The SIN conditions of experiment I and II were combined and analyzed as a single stimulus condition. However, two contrasts were included to investigate the effect of modulation type by comparing SAM vs. SIN, to represent experiment I, and MM vs. SIN, to represent experiment II. Significance levels were set at $\alpha=0.05$, and in case of multiple comparisons, $p$-values were corrected based on Holm's method. To obtain an estimate of the final loudness adaptation percentage 
of each stimulus condition, the data of each stimulus condition was fitted using polynomials. A linear polynomial was used for the basic fit, with second-order and third-order polynomials being used if they led to a better fit.

\section{RESULTS}

\section{Estimated final loudness adaptation percentages}

The results of the loudness adaptation experiments are shown in Figure 2. Based on visual inspection, loudness adaptation was only found at $30 \mathrm{~dB}$ SL, with more adaptation occurring at $2000 \mathrm{~Hz}$. Most of the adaptation occurred in the first 150 s. At $30 \mathrm{~dB}$ SL and $2000 \mathrm{~Hz}$, the final estimated loudness adaptation percentages, based on the fitted polynomials, were $-65.8 \%,-53.6 \%$, and $-49.8 \%$ for the MM, SIN, and SAM modulation types respectively. This difference in loudness adaptation percentage between the three modulation types was more pronounced at $30 \mathrm{~dB}$ SL and $500 \mathrm{~Hz}$, with estimated loudness adaptation percentages of $45.2 \%,-38.9 \%$, and $-10.9 \%$ for the MM, SIN, and SAM modulation types respectively.

In contrast, at $70 \mathrm{~dB}$ SL, small amounts of loudness enhancement were only found for the $500 \mathrm{~Hz}$ stimulus conditions. The final estimated loudness adaptation percentages were $9.2 \%, 13.7 \%$, and $5.4 \%$ at $500 \mathrm{~Hz}$, and $-0.1 \%,-1.8 \%$, and $-5.1 \%$ at $2000 \mathrm{~Hz}$ for the MM, SIN, and SAM modulation types respectively.

\section{Predictors of loudness adaptation}

A summary of the linear mixed-effects model is shown in Table 1. Significant main effects were found for time, threshold, sensation level, carrier frequency, and the modulation contrast SAM vs. SIN. The modulation contrast MM vs. SIN was not significant. However, several interaction effects were found, and are described in the following sections.

\section{Interaction effects between carrier frequency, modulation type, and sensation level}

The interaction effects between carrier frequency, modulation type, and sensation level are shown in Figure 3 A-C. 
Figure $3 \mathrm{~A}$ shows the significant interaction effect between sensation level and carrier frequency $(b=0.06, t(3127)=3.48, p=0.001$, see Table 1$)$. At $70 \mathrm{~dB}$ SL, similar loudness adaptation percentages around 0\% were found. In contrast, at $30 \mathrm{~dB}$ SL, more loudness adaptation was found for $2000 \mathrm{~Hz}$ compared to $500 \mathrm{~Hz}$.

Figure $3 \mathrm{~B}$ shows the interaction effect between modulation type and sensation level. Only the interaction effect with the modulation contrast SAM vs. SIN was significant $(b=-0.07, t(3127)=-2.49, p=0.013$, see Table 1$)$, which means that a significant difference in the slope of the SIN-SAM line was found between $30 \mathrm{~dB}$ SL and $70 \mathrm{~dB}$ SL. The p-value of the interaction effect with the modulation contrast MM vs. SIN was equal to 0.05. At $30 \mathrm{~dB}$ SL, MM stimuli showed more loudness adaptation than SIN stimuli, which in turn showed more loudness adaptation than SAM stimuli.

Figure $3 \mathrm{C}$ shows the significant interaction effects between modulation type and carrier frequency $(b=-0.12, t(3127)=-5.50, p<0.001$ for the SAM vs. SIN contrast, and $b=-0.06, t(3127)=-2.60, p=0.009$ for the MM vs. SIN contrast, see Table 1). The difference between the modulation types was especially apparent at a carrier frequency of $500 \mathrm{~Hz}$, with a more pronounced difference in slope for the SAM vs. SIN contrast.

Furthermore, only for the modulation contrast SAM vs. SIN, a significant interaction effect was found between sensation level, modulation type, and carrier frequency. Post-hoc analyses revealed no significant effects of time on loudness adaptation percentages, i.e. no loudness adaptation, for the $70 \mathrm{~dB}$ SL stimulus conditions and the SAM-500-30 stimulus condition (see Table 2).

In summary, while loudness adaptation only occurred at $30 \mathrm{~dB}$ SL and was observed for both carrier frequencies, significantly more loudness adaptation occurred at $2000 \mathrm{~Hz}$. A difference was also found between modulation types, with more loudness adaptation for MM stimuli compared to SIN stimuli, and also more loudness adaptation for SIN stimuli compared to SAM stimuli. This difference between modulation types was more pronounced at $500 \mathrm{~Hz}$ and $30 \mathrm{~dB}$ 
SL, with a disappearance of loudness adaptation for the SAM-500-30 stimulus condition.

\section{Interaction effects between threshold and other predictors}

The participants of experiment I had average thresholds of $7.4 \mathrm{~dB}$ SPL with a standard deviation of 5.9, and the participants of experiment II had average thresholds of 5.6 dB SPL with a standard deviation of $4.7 \mathrm{~dB}$ SPL.

As seen in Table 1, a significant interaction effect was found between the participants' threshold and the sensation level (see Figure 4). Only at $30 \mathrm{~dB}$ SL, the threshold had an influence on the loudness adaptation percentage, with lower thresholds leading to more loudness adaptation. Only one participant with a threshold of $19 \mathrm{~dB}$ SPL for the SIN-2000 stimulus had a deviant behavior. However, based on visual inspection, the adaptive staircase of this participant showed an unusual pattern. First, the staircase decreased to $5 \mathrm{~dB}$ SPL, with the lowest correctly detected threshold being $10 \mathrm{~dB}$ SPL. But thereafter the staircase increased again leading to a final estimated threshold of $19 \mathrm{~dB}$ SPL calculated as the mean of the last of 4 reversals. The participant might have been tired during the last trials and have had a lower true threshold.

The effect of threshold on loudness adaptation differed per modulation type, carrier frequency, and per combination of modulation and carrier frequency (see Table 1). In addition, the interaction effects between threshold, sensation level, modulation contrast, and carrier frequency indicate that the effect of threshold on loudness adaptation was different per stimulus condition. Therefore, correlation coefficients between the participant's thresholds and loudness adaptation percentages were calculated per stimulus condition. Moderate, but significant correlations were found for all stimulus conditions, except for the SAM-500-30 stimulus condition (see Table 3). In these calculations, all loudness adaptation percentages over time were included. The interaction effect between loudness adaptation percentage and threshold per stimulus condition is also shown in Figure 5. In the figure, each dot represents the average loudness adaptation 
percentage corresponding to a certain threshold for one stimulus condition, with different colors showing different stimulus conditions.

In summary, although the stimuli were presented to each participant at the same sensation level, the participant's threshold and thus the stimulation level in $\mathrm{dB}$ SPL seemed to have an effect on the loudness adaptation percentage at $30 \mathrm{~dB}$ SL. Significant effects were found for all stimulus conditions at $30 \mathrm{~dB}$ SL, except for the SAM-500-30 stimulus condition.

\section{DISCUSSION}

\section{The effects of carrier frequency, modulation type, and sensation level}

In this study, we demonstrated that both sinusoidally amplitude-modulated and mixed-modulated sinusoids can cause loudness adaptation. However, differences in loudness behavior across modulation types were also found at $30 \mathrm{~dB}$ SL. MM stimuli gave rise to more loudness adaptation than SIN stimuli, which in turn gave rise to more loudness adaptation than SAM stimuli. A more pronounced difference between SIN and SAM modulation was found at $500 \mathrm{~Hz}$, with a disappearance of loudness adaptation for the SAM-500-30 stimulus condition.

Loudness adaptation only occurred at the low sensation level of $30 \mathrm{~dB}$ SL. At the high sensation level of $70 \mathrm{~dB}$ SL, the same loudness behavior was found for MM, SIN, and SAM stimuli, with loudness enhancement percentages only present at the low carrier frequency of $500 \mathrm{~Hz}$. Our results also showed more loudness adaptation for a higher carrier frequency, since all modulation types showed more loudness adaptation at $2000 \mathrm{~Hz}$ compared to $500 \mathrm{~Hz}$. Furthermore, most of the loudness adaptation occurred in the first 100-150 s after stimulus onset. Thus, the results of this study are in agreement with many studies, e.g. [2], [6], [15]-[17], who also found more loudness adaptation at low sensation levels, high carrier frequencies, and in the first instants after stimulus onset.

The magnitude of adaptation was comparable to the results found in literature. Most similar to our stimuli are the $400 \mathrm{~Hz}$ and $2000 \mathrm{~Hz}$ pure tone stimuli presented at $30 \mathrm{~dB}$ SL used by Hellman et al. [17]. The final loudness adaptation 
percentages for these stimuli after $367 \mathrm{~s}$ were $-30 \%$ for both carrier frequencies. Although for these frequencies the percentages were similar; overall they found a progressive increase in adaptation with frequency. Tang et al. [6] described loudness enhancement effects at high sensation levels. Final estimated loudness adaptation percentages were $9.1 \%, 11.7 \%$, and $6.7 \%$ for unmodulated sinusoids with carrier frequencies of 125,1000 , and $2000 \mathrm{~Hz}$ respectively, all presented at $90 \mathrm{~dB}$ SPL. The results are comparable with this study, although we only found loudness enhancement at low carrier frequencies.

In the literature, simple loudness adaptation has mainly been investigated with unmodulated sinusoids. Scharf [2] found the same loudness adaptation percentages for frequency-modulated stimuli as for their unmodulated counterparts. For amplitude-modulated stimuli, he found an increase in loudness adaptation when increasing the frequency separation of two tones centered on $4000 \mathrm{~Hz}$. The loudness adaptation percentage saturated once the frequency separation reached the auditory filter bandwidth, and approached that of an unmodulated high-frequency tone. However, the final loudness adaptation percentage found by Scharf for frequency separations equal to or slightly larger than the auditory filter bandwidth was still slightly smaller compared to the final loudness adaptation percentage of the unmodulated high-frequency tone. Based on his findings, Scharf hypothesized that a change in excitation level within the auditory filter bandwidth in the cochlea can reduce or even eliminate loudness adaptation.

In our study, the different modulation types also yielded differences in stimulus bandwidths (see Figure 1). For SAM and MM stimuli, the bandwidth was $80 \mathrm{~Hz}$ and $240 \mathrm{~Hz}$ respectively. The auditory filter bandwidths for a center frequency of $500 \mathrm{~Hz}$ and $2000 \mathrm{~Hz}$ are $79 \mathrm{~Hz}$ and $241 \mathrm{~Hz}$, respectively [20], and are indicated in gray in Figure 1. Based on Scharf's hypothesis, we would expect reduced loudness adaptation percentages when there is amplitude modulation within the auditory filter bandwidth. For bandwidths equal to or slightly larger or smaller than the auditory filter bandwidth, we would expect similar loudness adaptation 
percentages or slightly reduced loudness adaptation percentages compared to their unmodulated counterparts.

First, for the MM-2000-30 and the SAM-500-30 stimulus conditions, with about equal bandwidths as the auditory filter bandwidths, we would expect the same or a slightly reduced amount of loudness adaptation as compared to their unmodulated counterparts. Indeed, the differences were small in the case of the MM-2000-30 condition. A statistically significant reduction in adaptation was found for the SAM-500-30 stimulus condition compared to the SIN-500-30 stimulus condition.

Second, for the MM-500-30 stimulus condition, with a bandwidth larger than the auditory filter bandwidth, we would also expect the same loudness adaptation percentage or a slightly reduced loudness adaptation percentage compared to the SIN-500-30 stimulus condition. Although the difference between MM-500-30 and SIN-500-30 was small, we found slightly more loudness adaptation for MM-50030 than for SIN-500-30 (see also figures 2 and 3).

Third, for the SAM-2000-30 stimulus, with a bandwidth clearly smaller than the auditory filter bandwidth, we would expect a significant reduction in loudness adaptation. However, the SAM-2000-30 and SIN-2000-30 stimulus condition showed similar final loudness adaptation percentages. Our statistical analysis also showed only a small difference in modulation types at $2000 \mathrm{~Hz}$. Interestingly, Scharf presented loudness adaptation percentages measured after 120s. As shown in Figure 2 in this study, a reduction in loudness adaptation percentages in the first 150s after stimulus onset was also found in this study. It seems that after around 150s, the amplitude modulations could no longer prevent loudness adaptation to occur.

Clearly, a dominant determinant for loudness adaptation to occur is the carrier frequency. Due to the tonotopic organization in the cochlea, more spread of excitation occurs for stimuli with low carrier frequencies compared to high carrier frequencies. This difference in spread of excitation in the cochlea might explain the difference in the reduction of loudness adaptation between SAM-500-30 and 
SAM-2000-30 stimuli. The latter would also give further support to the restricted excitation pattern hypothesis, which many studies in literature already support (e.g., [2], [16], [17]). According to this hypothesis, loudness adaptation only takes place if the excitation pattern in the cochlea is spatially restricted and also temporally steady. The studies supporting the hypothesis always found loudness adaptation for specific stimulus parameters that lead to a narrow and steady excitation pattern in the cochlea, such as low sensation levels, high carrier frequencies, and pure tones [5], [21]-[23]. Compared to SAM stimuli, MM stimuli are spatially restricted, but the temporal changes in the excitation pattern might have been too fast to lead to a reduction in loudness adaptation.

Recently, Wynne et al. [12], also found reduced loudness adaptation for amplitude-modulated stimuli. A much higher carrier frequency of $12 \mathrm{kHz}$ was used for all stimuli, which had a modulation frequency of 4, 20, or $100 \mathrm{~Hz}$ and were presented at $15 \mathrm{~dB}$ SL. Consequently, the bandwidths of these stimuli were always smaller than the auditory filter bandwidth, which is $1320 \mathrm{~Hz}$ for an unmodulated stimulus of $12 \mathrm{kHz}$. Regardless of the modulation frequency, the amplitude-modulated stimuli always showed a reduction in loudness adaptation after 180s, which corresponds to Scharf's hypothesis.

Wynne et al. proposed that reduced loudness adaptation might be due to an increased neural synchronization to amplitude modulations. Indeed, neural synchronization to amplitude-modulated stimuli is increased for auditory nerve fibers with higher characteristic frequencies [24]. However, a reduced loudness adaptation percentage was not found for mixed-modulated stimuli in this study, whereas MM stimuli evoke significantly larger ASSR amplitudes than SAM stimuli. This may indicate an increased synchronization somewhere on the auditory processing pathway for MM stimuli compared to SAM stimuli [25].

The differences in loudness adaptation between SAM and MM stimuli might be related to peripheral factors, central factors, or both. The role of central factors might be related to the observed difference between the processing of AM and FM stimuli in the auditory pathway up to the level of the supratemporal auditory 
cortex (e.g., [26]). Further evidence for possible central mechanisms involved in loudness adaptation was also described by Tang et al. [6].

The finding that loudness adaptation occurs when using certain modulated stimuli, might have an impact on ASSR measurements in clinical and research practice, in which these stimuli are often used. Namely, it is possible that the ASSRs evoked by these modulated stimuli will suffer a decrease in amplitude over time. ASSRs evoked at low intensities, such as during the objective estimation of hearing thresholds, would most likely be influenced by adaptation. More research is needed to define whether loudness adaptation effects need to be considered in the ASSR and if so, to what extent this has clinical implications. If the ASSR amplitude is shown to be related with loudness adaptation, it might be better to use SAM stimuli instead of MM stimuli at low sensation levels and low frequencies.

\section{The effect of threshold on loudness adaptation percentage}

We also investigated whether the participants' thresholds had a significant influence on their loudness adaptation percentages. We found that thresholds only had a significant effect on the loudness adaptation percentages at $30 \mathrm{~dB}$ SL. Moderate, but significant correlations between the participants' thresholds and their amount of loudness adaptation were found for all stimulus conditions at 30 $\mathrm{dB}$ SL, with the exception of the SAM-500-30 stimulus condition, in which also no significant loudness adaptation was found. Participants with higher thresholds tended to show less loudness adaptation.

A moderate correlation between thresholds and loudness adaptation was also described by Scharf [2], although unclear results were reported. In a study including 39 participants with thresholds between -14 and $42 \mathrm{~dB}$ SPL, he did not find a correlation between thresholds and loudness adaptation percentages for a $4000 \mathrm{~Hz}$ stimulus at $10 \mathrm{~dB}$ SL presented in free field. However, in a study including 54 participants whose thresholds fell between 2 and $15 \mathrm{~dB}$ SPL in 80\% of cases, he found a significant correlation of $r=0.36$, with the stimuli presented through earphones. The range of thresholds of the latter study is more similar to 
the range of this study. We found average thresholds of $7.4+-5.9 \mathrm{~dB} \mathrm{SPL}$, and $5.6+-4.7 \mathrm{~dB}$ SPL for experiment 1 and 2 respectively.

Thus, the thresholds of the participants or the stimulation level in dB SPL seems to play a crucial role in determining loudness adaptation. Differences in loudness adaptation percentage were found between participants, even when the sensation level was the same for all participants (30 dB SL). It would seem that the degree of adaptation is determined by absolute $\mathrm{dB}$ SPL values rather than sensation levels, meaning it would be better to use fixed dB SPL stimulation values instead of $\mathrm{dB}$ SL values in future loudness adaptation experiments, unless you want to differentiate between people. Intensities of around $40 \mathrm{~dB}$ SPL seem to be the limit for loudness adaptation for the stimulus conditions in this study (see Figure 5).

The occurrence of loudness adaptation at low sound pressure levels, irrespective of sensation level, might be related to the type of auditory nerve fiber activated. Auditory nerve fibers with a high spontaneous discharge rate (>20 sp/s) have lower thresholds for activation by acoustic stimulation than the auditory nerve fibers with low spontaneous discharge rates $(<20 \mathrm{sp} / \mathrm{s})$, and they also have a tendency to saturate within $30 \mathrm{~dB}$ of threshold [27], [28]. Loudness adaptation might mainly be caused by the high spontaneous discharge rate fibers. It has also been shown that auditory nerve fibers with low characteristic frequencies and high spontaneous discharge rates show the poorest envelope synchronizations for amplitude-modulated stimuli [24]. However, although neural adaptation (measuring over an interval of $192 \mathrm{~s}$ ) was found in nerve fibers with both low and high spontaneous discharge rates in a study of Javel [29] in cats, more adaptation was found for nerve fibers with low spontaneous discharge rates, which seems contradictory. However, this neural adaptation is not necessarily related to the loudness adaptation phenomenon we observe in humans, e.g., more neural adaptation was also found at high sensation levels. More research is needed to investigate the contribution of the auditory nerve fiber types or other underlying physiological mechanisms and their relation with perceptual loudness adaptation phenomena in humans. 


\section{CONCLUSION}

440 In this study, at $70 \mathrm{~dB}$ SL, small amounts of loudness enhancement were found

441 for all modulation types at $500 \mathrm{~Hz}$ only. At $30 \mathrm{~dB}$ SL, loudness adaptation was

442 found to occur for all modulation types, with more loudness adaptation at 2000 $\mathrm{Hz}$ compared to $500 \mathrm{~Hz}$. Mixed-modulated sinusoids showed more adaptation than unmodulated sinusoids, which in turn showed more adaptation than sinusoidally amplitude-modulated sinusoids. The difference between modulation types was more pronounced at $500 \mathrm{~Hz}$, with a disappearance of loudness adaptation for the sinusoidally amplitude-modulated stimulus condition at this carrier frequency. The fact that modulated stimuli can cause loudness adaptation might have implications for ASSR measurements, although this remains to be investigated. Furthermore, with the stimuli being presented at the same sensation levels to all participants, at $30 \mathrm{~dB}$ SL moderate but significant correlations were found between the participants' thresholds and their loudness adaptation percentages, with lower thresholds leading to more loudness 454 adaptation. 


\section{List of figure captions}

Figure 1: Frequency spectra of unmodulated sinusoids (SIN), sinusoidally amplitude-modulated sinusoids (SAM) and mixed-modulated sinusoids (MM). The dotted lines are the $500 \mathrm{~Hz}$ carrier stimulus conditions, the solid lines the $2000 \mathrm{~Hz}$ carrier stimulus conditions. The auditory filter bandwidths corresponding to each center frequency are represented by a gray shaded area.

Figure 2: The loudness adaptation percentages of the different stimulus conditions over time, shown per carrier frequency and sensation level. Unmodulated sinusoids (SIN) are indicated with dots, sinusoidally amplitudemodulated (SAM) conditions with crosses and mixed-modulated (MM) conditions with squares (and are blue, red and green respectively in the colored version). The SIN and MM data points are slightly shifted over the $\mathrm{x}$-axis in order to increase the clarity of the figure. The best fitting polynomials for each stimulus condition are plotted in the form of thin lines on top of the data. Error bars indicate standard errors of the mean.

Footnote: We changed one response of one participant, even though it was not removed after the MAD-median rule. The participant gave the following responses for the SIN-500-30 stimulus condition each 20 s: [5 55555558555555 5]. In loudness adaptation percentages, this leads

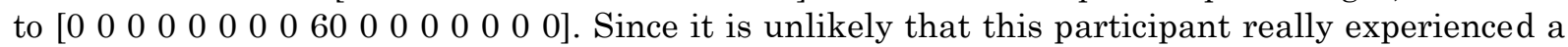
sudden enhancement of $60 \%$ in loudness, and we assume it might be a typing error (number 5 and number 8 are close to each other on a numeric keyboard), we decided to treat the " 8 " as an outlier, and removed it from further analysis.

Figure 3: The effects of sensation level, modulation type and carrier frequency on the loudness adaptation percentage. The mean loudness adaptation percentage indicates the mean of the loudness estimates over the specific times. Error bars indicate standard errors of the mean. The interaction effects are shown between (A) sensation level and carrier frequency, (B) modulation type and sensation level, and (C) modulation type and carrier frequency. The dashed line (blue in the colored version) indicates in (A) and (C) the $2000 \mathrm{~Hz}$ carrier frequency, and in (B) the $70 \mathrm{~dB}$ sensation level.

Figure 4: The effect of the participants' thresholds on the loudness adaptation percentages for 30 and $70 \mathrm{~dB}$ SL. Each dot represents the average loudness adaptation percentage over all time points, corresponding to a certain threshold. The blue triangles represent the $70 \mathrm{~dB}$ sensation level, and the red circles the 30 dB sensation level.

Figure 5: The effect of threshold on the loudness adaptation percentage split up per carrier frequency, and per modulation type (only shown for the $30 \mathrm{~dB}$ sensation level). Each dot represents the average loudness adaptation percentage over all time points, corresponding to a certain threshold. Different colors represent different stimulus conditions. The SIN, SAM, and MM stimulus 
conditions are plotted with blue squares, red triangles, and green circles respectively. 


\section{Tables and table captions}

Table 1: Statistical details of the best fitting model for loudness adaptation including the combined data of experiment I and II. Each predictor's t-value, pvalue, coefficient, and the 95\% confidence interval (CI) of the coefficient are shown. Abbreviations: Mod. SAM = modulation contrast SAM vs. SIN, Mod. MM = modulation contrast MM vs. SIN, Sens. level = sensation level, Freq. = carrier frequency.

\begin{tabular}{|c|c|c|c|c|c|}
\hline Predictor & t-value & p-value & Coefficient & \multicolumn{2}{|c|}{ 95\% CI } \\
\hline Intercept & -4.382 & $<0.001$ & -4.405 & -6.369 & -2.442 \\
\hline Time & -9.593 & $<0.001$ & -0.145 & -0.174 & -0.115 \\
\hline Time $\mathrm{x}$ Threshold & 6.070 & $<0.001$ & 0.007 & 0.004 & 0.009 \\
\hline Time x Sens. level & 16.356 & $<0.001$ & 0.212 & 0.187 & 0.238 \\
\hline Time x Mod. SAM & 5.939 & $<0.001$ & 0.107 & 0.072 & 0.142 \\
\hline Time x Mod. MM & -0.388 & 0.698 & -0.007 & -0.042 & 0.028 \\
\hline Time x Freq. & -11.283 & $<0.001$ & -0.122 & -0.144 & -0.101 \\
\hline Time x Sens. level x Freq. & 3.482 & 0.001 & 0.060 & 0.026 & 0.094 \\
\hline Time x Sens. level x Mod. SAM & -2.485 & 0.013 & -0.066 & -0.117 & -0.014 \\
\hline Time x Sens. level x Mod. MM & 1.942 & 0.052 & 0.048 & 0.000 & 0.096 \\
\hline Time x Mod. SAM x Freq. & -5.504 & $<0.001$ & -0.119 & -0.161 & -0.077 \\
\hline Time x Mod. MM x Freq. & -2.597 & 0.009 & -0.055 & -0.096 & -0.014 \\
\hline Time x Sens. level x Mod. SAM x Freq. & 2.772 & 0.006 & 0.095 & 0.028 & 0.163 \\
\hline Time x Sens. level x Mod. MM x Freq. & 0.579 & 0.563 & 0.017 & -0.041 & 0.076 \\
\hline Time $\mathrm{x}$ Threshold $\mathrm{x}$ Sens. level & -7.939 & $<0.001$ & -0.012 & -0.015 & -0.009 \\
\hline Time x Threshold x Freq. & 1.962 & 0.050 & 0.003 & $1 \mathrm{E}-05$ & 0.005 \\
\hline Time x Threshold x Mod. SAM & -2.843 & 0.004 & -0.005 & -0.009 & -0.002 \\
\hline Time $\mathrm{x}$ Threshold x Mod. MM & -1.577 & 0.115 & -0.003 & -0.007 & 0.001 \\
\hline Time x Threshold x Sens. level x Freq. & 1.597 & 0.110 & 0.003 & -0.001 & 0.007 \\
\hline Time x Threshold x Sens. level x Mod. SAM & 0.936 & 0.349 & 0.003 & -0.003 & 0.008 \\
\hline Time x Threshold x Sens. level x Mod. MM & 0.773 & 0.440 & 0.002 & -0.003 & 0.008 \\
\hline Time x Threshold x Mod. SAM x Freq. & 3.422 & 0.001 & 0.008 & 0.004 & 0.013 \\
\hline Time $\mathrm{x}$ Threshold $\mathrm{x}$ Mod. MM x Freq. & 6.898 & $<0.001$ & 0.019 & 0.014 & 0.025 \\
\hline Time x Threshold x Sens. level x Mod. SAM x Freq. & -2.288 & 0.022 & -0.008 & -0.015 & -0.001 \\
\hline Time x Threshold x Sens. level x Mod. MM x Freq. & -3.442 & 0.001 & -0.013 & -0.020 & -0.006 \\
\hline
\end{tabular}

Table 2: Post-hoc analyses for the effect of time on loudness adaptation percentages for each stimulus condition involved in the SAM vs. SIN modulation contrast. All loudness adaptation percentages, at each time point, were used. The predictor time's t-value, p-value (corrected based on Holm's method), coefficient, and the $95 \%$ confidence interval (CI) of the coefficient are shown.

\begin{tabular}{l|rrr|} 
Stimulus condition & t-value & p-value (Holm corrected) & Coefficient \\
\hline SAM-500-30 & -1.239 & 0.868 & -0.038 \\
SAM-2000-30 & -5.662 & $<0.001$ & -0.159 \\
SAM-500-70 & 1.397 & 0.822 & 0.033 \\
SAM-2000-70 & -0.084 & 0.933 & -0.002 \\
\hline SIN-500-30 & -6.334 & $<0.001$ & -0.123 \\
SIN-2000-30 & -8.183 & $<0.001$ & -0.132 \\
SIN-500-70 & 1.017 & 0.868 & 0.018 \\
SIN-2000-70 & -1.137 & 0.868 & -0.023 \\
\hline
\end{tabular}


Table 3: Correlation coefficients between the participants' thresholds and their loudness adaptation percentage per stimulus condition. All loudness adaptation percentages, at each time point, were used for the calculation of the correlation coefficients.

\begin{tabular}{l|rr|} 
Stimulus condition & Correlation coefficient $\boldsymbol{r}$ & p-value (Holm corrected) \\
\hline SAM-500-30 & 0.017 & 0.809 \\
SAM-2000-30 & 0.414 & $<0.001$ \\
\hline SIN-500-30 & 0.336 & $<0.001$ \\
SIN-2000-30 & 0.256 & $<0.001$ \\
\hline MM-500-30 & 0.408 & $<0.001$ \\
MM-2000-30 & 0.309 & $<0.001$ \\
\hline
\end{tabular}




\section{References}

[1] M. Florentine, A. N. Popper, and R. R. Fay, Loudness. New York: Springer, 2011.

[2] B. Scharf, "Loudness adaptation," in Hearing research and theory, J. V. Tobias and E. D. Schubert, Eds. New York: Academic Press, 1983, pp. 1-56.

[3] R. S. Schlauch, "A cognitive influence on the loudness of tones that change continuously in level," J. Acoust. Soc. Am., vol. 92, no. 2, pp. 758-765, 1992.

[4] G. Canévet and B. Scharf, "The loudness of sounds that increase and decrease continuously in level," J. Acoust. Soc. Am., vol. 88, no. 5, pp. 2136-2142, 1988.

[5] S. Meunier, "An overview of Bertram Scharf's research in France on loudness adaptation," in Proceedings of Meetings on Acoustics, 2-7 June, Montreal, Canada, 2013, vol. 19, pp. 1-7.

[6] Q. Tang, S. Liu, and F.-G. Zeng, "Loudness adaptation in acoustic and electric hearing," J. Assoc. Res. Otolaryngol., vol. 7, no. 1, pp. 59-70, 2006.

[7] T. W. Picton, Human auditory evoked potentials, 1st ed. San Diego: Plural Publishing Inc., 2011.

[8] T. W. Picton, M. S. John, A. Dimitrijevic, and D. Purcell, "Human auditory steadystate responses,” Int. J. Audiol., vol. 42, no. 4, pp. 177-219, 2003.

[9] H. Luts, "Diagnosis of hearing loss in newborns: clinical application of auditory steady-state responses," PhD thesis, KU Leuven, 2005.

[10] G. Rance, Auditory steady-state response: generation, recording and clinical applications. San Diego-Oxford-Brisbane: Plural Publishing Inc., 2008.

[11] M. S. John, A. Dimitrijevic, P. van Roon, and T. W. Picton, "Multiple auditory steady-state responses to AM and FM stimuli.," Audiol. Neurootol., vol. 6, no. 1, pp. 12-27, 2001.

[12] D. P. Wynne, S. E. George, and F.-G. Zeng, "Amplitude modulation reduces loudness adaptation to high-frequency tones," J. Acoust. Soc. Am., vol. 138, no. 1, pp. 279-283, 2015.

[13] T. Francart, A. van Wieringen, and J. Wouters, "APEX 3: a multi-purpose test platform for auditory psychophysical experiments," J. Neurosci. Methods, vol. 172, no. 2, pp. 283-293, 2008.

[14] A. Miskiewicz, R. P. Hellman, and B. Scharf, "Frequency and temporal effects in loudness adaptation," in 15th International Congress on Acoustics, 26-30 June, Trondheim, Norway, 1995, pp. 203-206.

[15] D. P. Wynne, F.-G. Zeng, S. Bhatt, H. J. Michalewski, A. Dimitrijevic, and A. Starr, "Loudness adaptation accompanying ribbon synapse and auditory nerve disorders," Brain, vol. 136, no. 5, pp. 1626-1638, 2013. 
[16] A. Miskiewicz, B. Scharf, R. Hellman, and C. Meiselman, "Loudness adaptation at high frequencies,” J. Acoust. Soc. Am., vol. 94, no. 3, pp. 1281-1286, 1993.

[17] R. Hellman, A. Miśkiewicz, and B. Scharf, "Loudness adaptation and excitation patterns: effects of frequency and level," J. Acoust. Soc. Am., vol. 101, no. 4, pp. 2176-2185, 1997.

[18] R. R. Wilcox, D. A. Granger, and F. Clark, "Modern robust statistical methods: basics with illustrations using psychobiological data," Univers. J. Psychol., vol. 1, no. 2 , pp. 21-31, 2013.

[19] R Core Team, "R: A language and environment for statistical computing." $R$ Foundation for Statistical Computing, Vienna, Austria, http://www.r-project.org/, 2014.

[20] B. R. Glasberg and B. C. J. Moore, "Derivation of auditory filter shapes from notched-noise data," Hear. Res., vol. 47, no. 1-2, pp. 103-138, 1990.

[21] J. M. Snyder, "Threshold adaptation in normal listeners activated," J. Acoust. Soc. Am., vol. 53, no. 2, pp. 435-439, 1972.

[22] R. Carhart, "Clinical determination of abnormal auditory adaptation," AMA. Arch. Otolaryngol., vol. 65, no. 1, pp. 32-39, 1957.

[23] W. O. Olsen and D. Noffsinger, "Comparison of one new and three old tests of auditory adaptation," Arch. Otolaryngol., vol. 99, no. 2, pp. 94-99, 1974.

[24] P. X. Joris and T. C. T. Yin, "Responses to amplitude-modulated tones in the auditory nerve of the cat," J. Acoust. Soc. Am., vol. 91, no. 1, pp. 215-232, 1992.

[25] L. T. Cohen, F. W. Rickards, and G. M. Clark, "A comparison of steady-state evoked potentials to modulated tones in awake and sleeping humans.," J. Acoust. Soc. Am., vol. 90, no. 5, pp. 2467-2479, 1991.

[26] J. P. Mäkelä, R. Hari, and A. Linnankivi, "Different analysis of frequency and amplitude modulations of a continuous tone in the human auditory cortex: a neuromagnetic study.," Hear. Res., vol. 27, no. 3, pp. 257-264, 1987.

[27] A. C. Furman, S. G. Kujawa, and M. C. Liberman, "Noise-induced cochlear neuropathy is selective for fibers with low spontaneous rates.," J. Neurophysiol., vol. 110, no. 3, pp. 577-586, 2013.

[28] I. M. Winter, D. Robertson, and G. K. Yates, "Diversity of characteristic frequency rate-intensity functions in guinea pig auditory nerve fibres.," Hear. Res., vol. 45, no. 3, pp. 191-202, 1990.

[29] E. Javel, "Long-term adaptation in cat auditory-nerve fiber responses.," J. Acoust. Soc. Am., vol. 99, no. 2, pp. 1040-1052, 1996. 


\section{Acknowledgements}

The authors thank all participants. Special thanks to Astrid Buelens, Roosmarij Clercx, and Steffi Tilkens for their help in acquiring the data.

Maaike Van Eeckhoutte was supported by a PhD-grant for Strategic Basic Research by the Agency for Innovation by Science and Technology in Flanders (IWT, 131106).

Address for correspondence: Maaike Van Eeckhoutte, KU Leuven, ExpORL, Department of Neurosciences, Herestraat 49, box 721, B-3000 Leuven, Belgium.

E-mail: maaike.vaneeckhoutte@med.kuleuven.be

Fax number: +32 16330486

Telephone number: +3216330488 
Figure 1

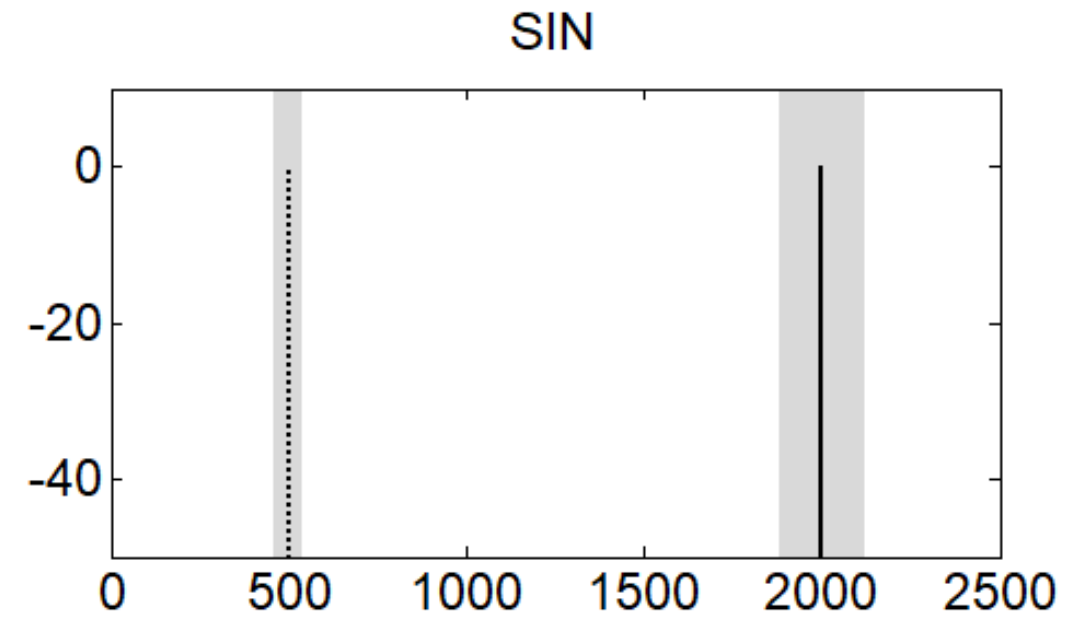

SAM

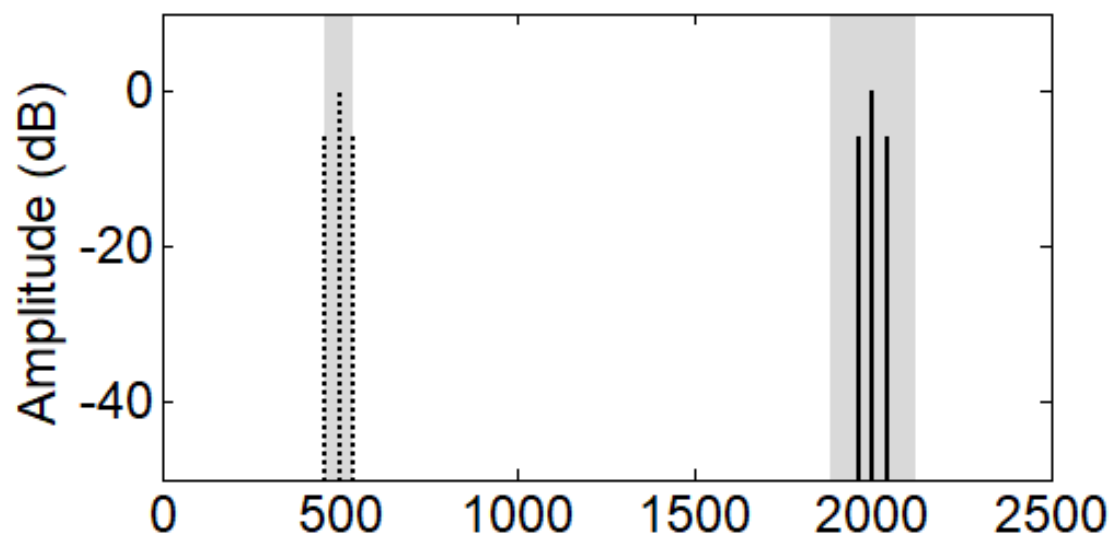

MM

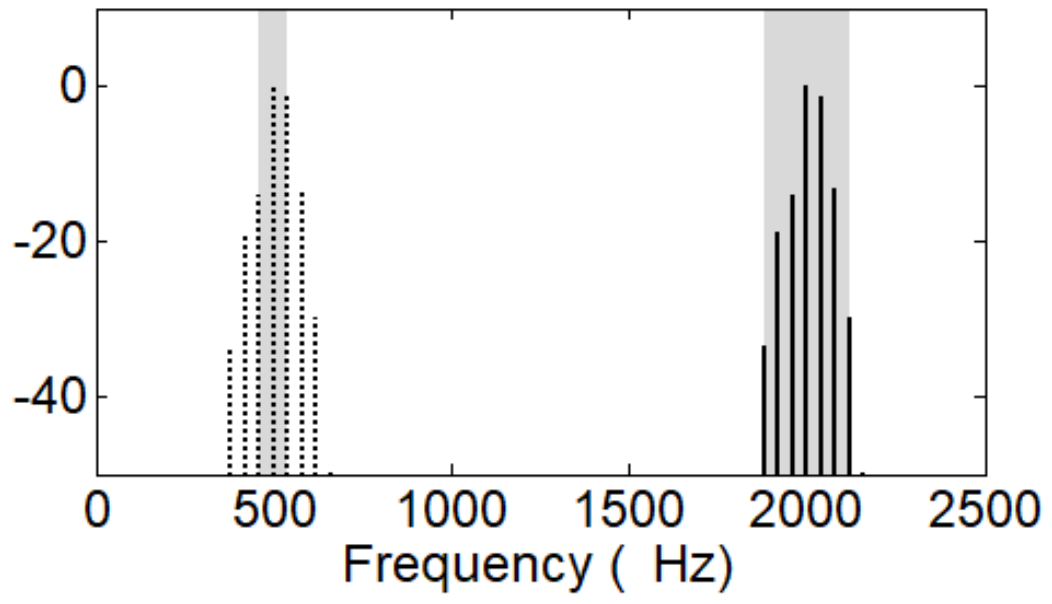


Figure 2

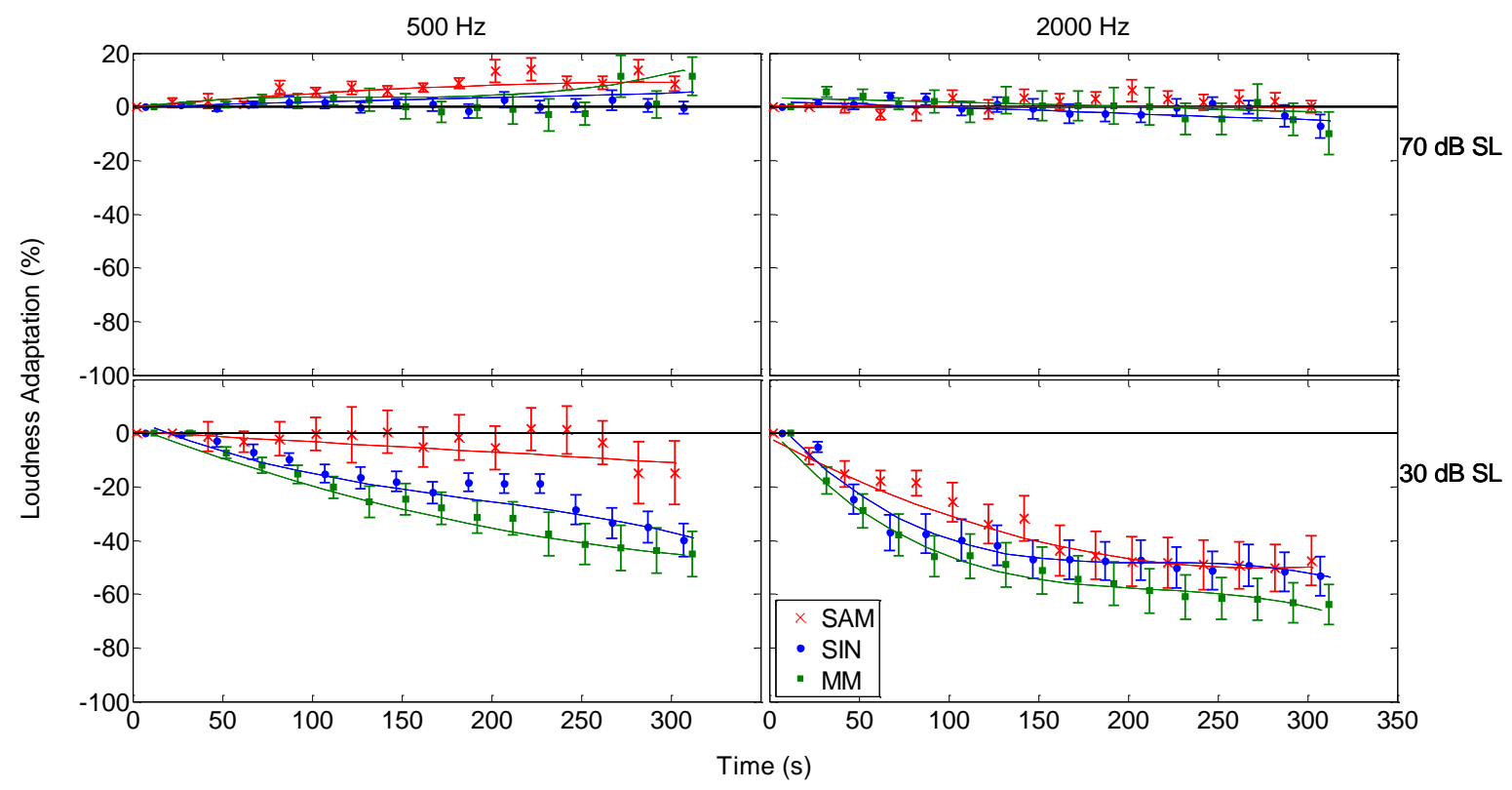


Figure 3

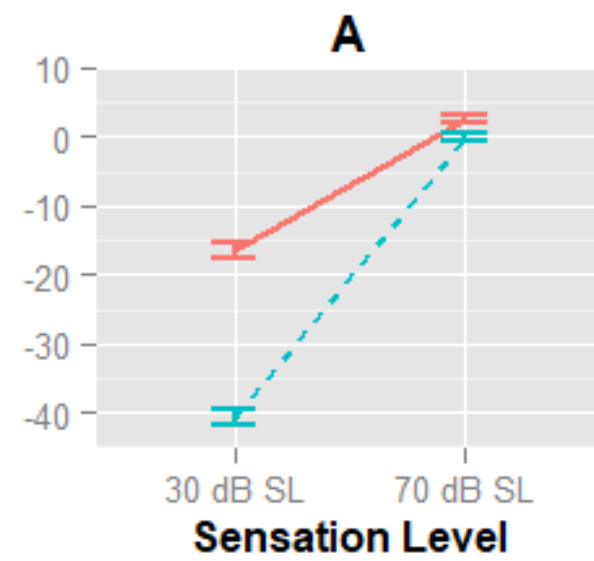

\author{
Frequency \\ $-500 \mathrm{~Hz}$ \\ $-2000 \mathrm{~Hz}$
}

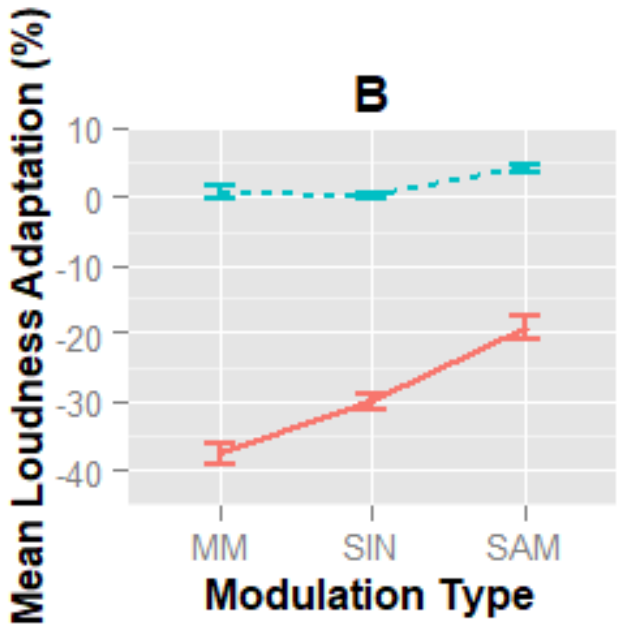

Level

$-30 \mathrm{~dB}$ SL

$-70 \mathrm{~dB} S \mathrm{SL}$

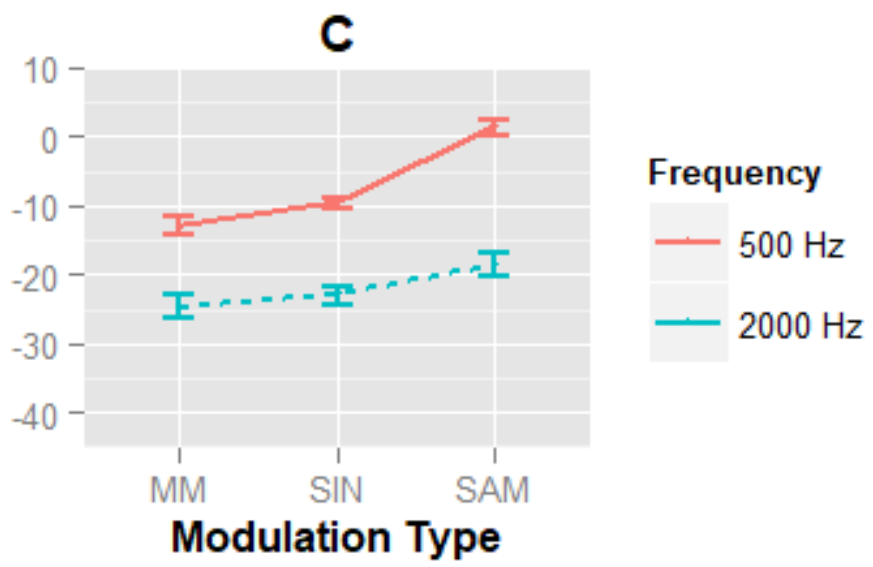


Figure 4

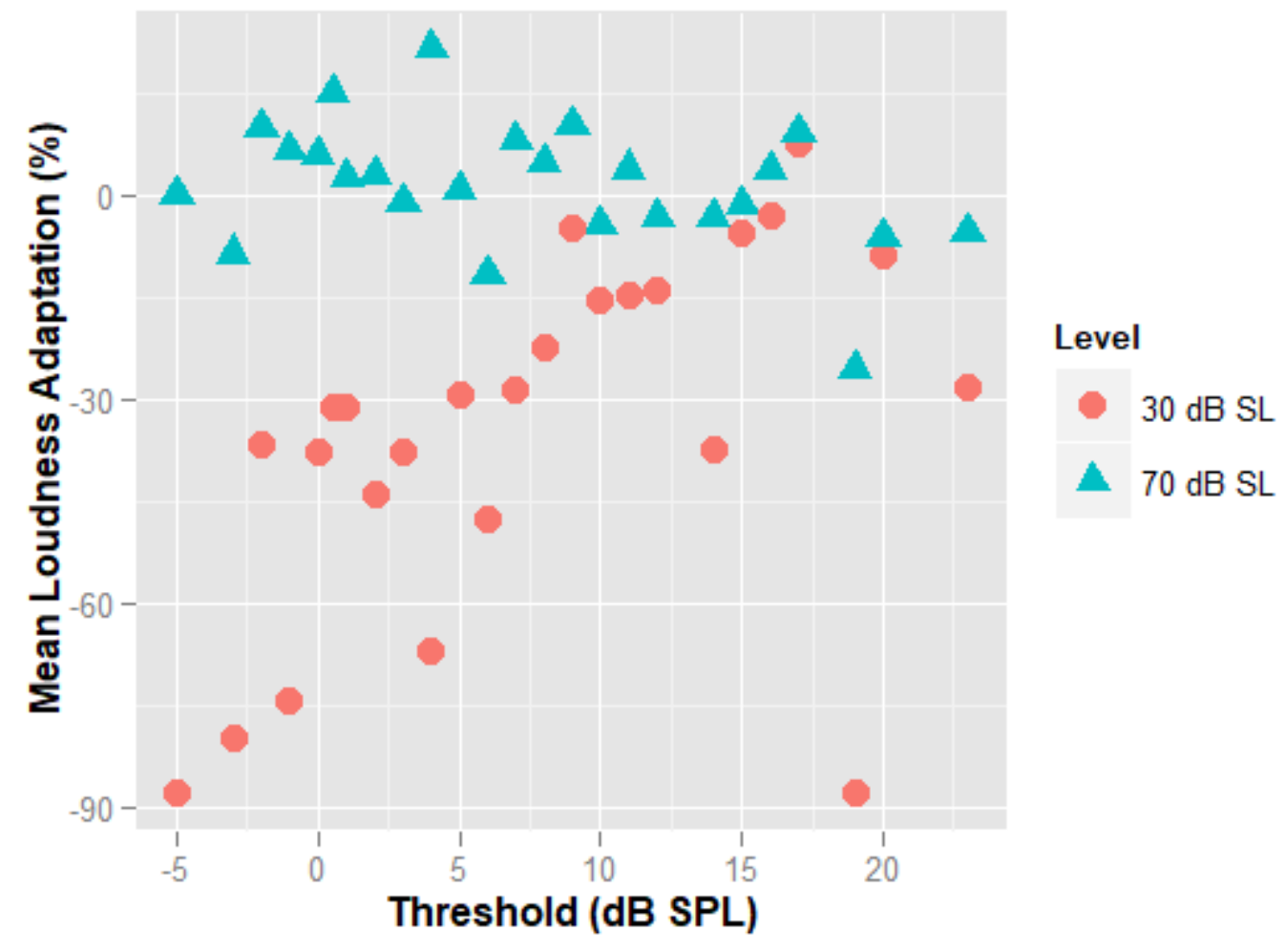


Figure 5

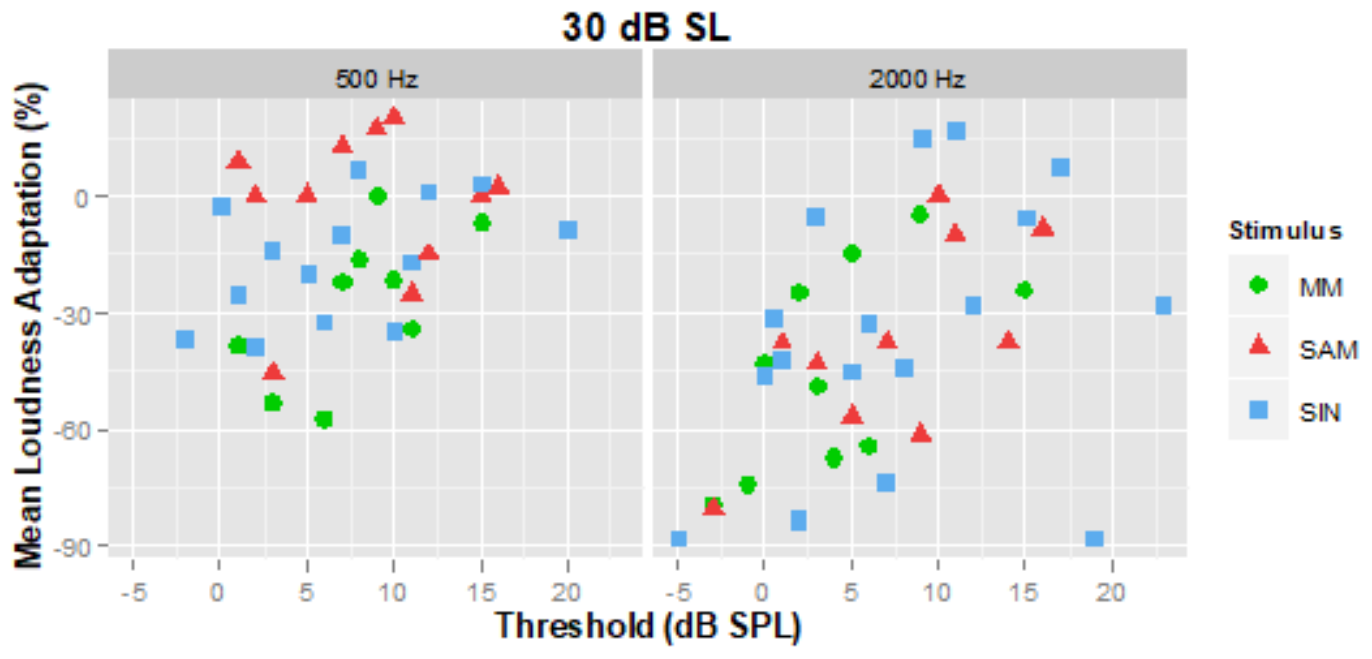

